\title{
COMMENT: ON CLOSING OF ENGLISH JOURNAL OF JSCS AND THE BIRTH OF NEW JOURNAL JJSD
}

\author{
Yutaka Tanaka*
}

As announced by the current editors Wataru Sakamoto and Hiroshi Yadohisa, the English journal of JSCS "Journal of the Japanese Society of Computational Statistics" (JJSCS) closes at this issue (Volume 30, Number 2), and is going to join a new journal "Japanese Journal of Statistics and Data Science" (JJSD) that will start in 2018 as an official journal of the Japanese Federation of Statistical Science Associations (JFSSA). All of six member societies will jointly contribute to the publication of JJSD.

JSCS was established in 1986. I played the role of chief editor of JJSCS since then until 1990, and was responsible for the publication of volumes 1 to 3 . The major purpose of establishing JSCS was to make a progress in computational statistics in Japan by providing a forum for people working in various aspects of computational statistics, e.g., statisticians engaged in the research and application of statistical theory and methods, computer scientists/engineers engaged in the development of statistical software, and statisticians/data analysts engaged in the analysis of data obtained with surveys, experiments or other means, and provide them opportunities for exchanging information and ideas and, if possible, for finding seeds for joint works among them. For producing better products as well as for getting more valuable outcomes using the products, it is vital to know what the counterpart really wishes between the producers and the users (or consumers) of everything including statistical methods and software.

In those days our major interest was in the $\mathrm{R} \& \mathrm{D}$ of statistical program packages (SPP) as described in President Address (JSCS Japanese journal, Volume 1, Number 1) by the first president Chooichiro Asano. I myself was interested in R \& D of SPP on personal computers with my colleagues, that is useful both for the data analysis and for the research work of statistical methods.

There are three remarkable traditions in JSCS. I like these traditions and think that they have contributed very much to the achievement of the purpose of establishment mentioned above. One is social gathering at the time of scientific meeting. Every time it is organized in such a way that participants can talk freely in a comfortable atmosphere for exchanging information and ideas. The second one is the tradition that president of JSCS is elected alternately from academic and non-academic communities so that both sides will be satisfied equally in a long period. The third one is that it is very positive in international activities, for example, Japan and Korea Conferences, Japan and China Symposia, and also organizing international meetings such as $25^{\text {th }}$ JSCS Symposium in Busan in 2011, two international scientific meetings for $30^{\text {th }}$ Anniversary in Okinawa and Seattle in 2015 and 2016.

Since around a decade ago the environment of statistics has been changing due to advances in the computer and information technology, and accordingly it has strong effect on statistical science especially computational statistics. Let us briefly review what happened.

*Professor Emeritus of Statistics, Okayama University, 1-1-1 Tsushima-naka, Kita-ku, Okayama 700-8530, Japan E-mail: ytanaka@ems.okayama-u.ac.jp 
In traditional statistics the data is usually obtained with experiment or survey. There appeared different types of unstructured massive data. They are obtained in scientific research and business, etc., for example, research in genomics, environmental science, medical science and meteorology, and in business activities to extract useful information on the web, POS, IoT, etc. They are called "Big Data". It is characterized by $3 \mathrm{Vs}$, i.e., (1) volume, (2) variety and (3) velocity, where velocity means how fast the data is generated and processed to meet the demands, or sometimes by $4 \mathrm{Vs}$ with additional (4) veracity, which means the quality or accurateness of the data. "Data Scientists" receive attention as professionals to derive valuable insights from such data. Ideally it is required for data scientists to have (1) programming skills for processing structured and unstructured data, (2) analytical skills for using appropriate methods in statistics and machine learning, and (3) business skills for understanding the problems to be solved as well as for deriving business insights from the results of analysis and reporting with appropriate visualization techniques to decision makers. Talent with the second category of skills is called deep analytical talent in MGI report mentioned below. Expression of the third category is for business application, but it will be easy to transform the expression for applications in science.

Hal Varian, the chief economist at Google, told in 2009, "The sexy job in the next 10 years will be statisticians", and McKinsey Global Institute (MGI) published a report on "Big Data: The next frontier for innovation, competition, and productivity" in 2011.The report says that much of modern economic activity, innovation, and growth could not take place without data, and it estimates precisely the potential annual value Big Data can create to be 300 billion dollars to US health care and 250 billion euros to Europe's Public sector administration. On the other hand it predicts there would be shortage of deep analytic talent to take advantage of big data in the US and other countries. In 2012 T. H. Davenport and D. J. Patil wrote in Harvard Business Review an article titled "Data Scientist: The Sexiest Job of the 21th Century". By the way, the opportunities to meet the term "Data Science" increased rapidly in recent years, but, it was not a new term for statisticians. It was used at the 2nd Japanese-French Scientific Seminar (organized by Yves Escoufier, Chikio Hayashi, and others) held in Montpellier. The title of the Seminar and the Proceedings published later by Academic Press in 1995 was "Data Science and Its Applications". This term was used for "new science with data at its core". The meaning is essentially the same to that currently used, though types of data to be analyzed have changed in a quarter century.

In 2012 US government announced a "Big Data R \& D Initiative". It promises to help to solve some Nation's pressing challenges, and to transfer the ability to use big data for scientific discovery, environmental and biomedical research, education, and national security. For this purpose the government invests more than $\$ 200$ million, and six organizations of the government announced their plans. Among them, National Science Foundation (NSF) encouraged research universities to develop interdisciplinary graduate programs to prepare the next generation of data scientists and engineers.

ASA News on October, 2015 contained an impressive statement of ASA president D. R. Morganstein as follows: "We're entering an era of tremendous growth in the profession of statistics that is not unlike the growth of computing professionals in the 1960s and 1970s. At that time, software engineers and programmers were still relatively rare, but grew rapidly and steadily as computing became something every large company needed to remain competitive. We're seeing similar trajectory in statistics. Advances in computing, technology and Big Data continue to raise the demand for statisticians." EU announced BIG (Big Data Public Private Forum) 28 months with 2 million euro and succeeding Horizon 2020 
(2014-2020), with 900 million euro investment. Now many institutes, departments or graduate programs have been established in universities or research organizations in the US and European countries.

Also in Japan statistics attracted a good deal of public attention in 2013. An enlightening book on statistics was sold more than 400 thousand copies. It was a quite exceptional event. Special issue on statistics was published in several weekly or monthly magazines such as Weekly Economist and Weekly Diamond in the same year. Two proposals were presented in 2014 on Research and Education of Statistical Science in the Era of Big Data and related topics by Committee of Mathematical Science and Committee of Information Science in the Science Council of Japan.

In 2016 the Ministry of Education, Culture, Sports, Science and Technology assigned six universities to play central roles for promoting the education of Mathematics and Data Science to foster talent needed in the Era of Big Data. For the first time in Japan Faculty of Data Science was established in Shiga University in 2017, and School of Data Science will be established in Yokohama City University in 2018. Education program for data scientists has been made also in many other universities. Now there are quite a number of statisticians engaged in the analysis of big data in genomics, bioinformatics, environment science, sports and so on, and they are going to get interesting results. As such activities increase, potential values as described in MGI report become reality.

The demand for statisticians and data scientists is clearly growing in US, Europe, Japan and other countries. I was deeply impressed by the statement of Morganstein, as I myself had similar experiences as a computing professional at a pharmaceutical company in 1960s and 1970s. I believe that we are now in the era of tremendous growth in the profession of statistics, though there may be differences between US and Japan. The appearance of faculty of data science is a breakthrough in the history of statistical education in Japan. It might be the result of the above mentioned (and related other) activities. I greatly appreciate the efforts of people who have contributed to those activities, and expect further improvement of the system of statistical research and education.

It was a good decision for JSCS to jointly contribute with other member societies to the publication of JJSD. JJSD and related activities will encourage those who are now engaged in research, education and applications of statistical science (including data science) and who are now studying to be next generation statisticians or data scientists. There will be no problem for closing JJSCS for JSCS members, because its main role has been to publish research papers of computational statistics in English. In the future, new journal JJSD, in which JJSCS revives, is going to play the same role for JSCS members. JJSD will have advantages such that it covers wider fields in statistics and has much larger number of contributors and readers. I hope that JJSD will be successful as an international journal for statisticians not only in Japan and neighboring countries but in the world.

(Received: February 1, 2018) 
